Article

\title{
Subwavelength Grating Double Slot Waveguide Racetrack Ring Resonator for Refractive Index Sensing Application
}

\author{
Nikolay Lvovich Kazanskiy ${ }^{1,2}$, Svetlana Nikolaevna Khonina ${ }^{1,2}$ and Muhammad Ali Butt ${ }^{1, * \text { D }}$ \\ 1 Department of Technical Cybernetics, Samara National Research University, 443086 Samara, Russia; \\ kazansky@smr.ru (N.L.K.); khonina@smr.ru (S.N.K.) \\ 2 Institute of RAS-Branch of the FSRC “Crystallography and Photonics" RAS, 443086 Samara, Russia \\ * Correspondence: ali_ciit_engineer@yahoo.com
}

Received: 24 May 2020; Accepted: 15 June 2020; Published: 17 June 2020

\begin{abstract}
In this paper, a racetrack ring resonator design based on a subwavelength grating double slot waveguide is presented. The proposed waveguide scheme is capable of confining the transverse electric field in the slots and the gaps between the grating segments. This configuration facilitates a large light-matter interaction which elevates the sensitivity of the device approximately 2.5 times higher than the one that can be obtained via a standard slot waveguide resonator. The best sensitivity of the design is obtained at $1000 \mathrm{~nm} / \mathrm{RIU}$ by utilizing a subwavelength grating double slot waveguide of period $300 \mathrm{~nm}$. The numerical study is conducted via 2D and 3D finite element methods. We believe that the proposed sensor design can play an important role in the realization of highly sensitive lab-on-chip sensors.
\end{abstract}

Keywords: single-slot waveguide; double slot waveguide; subwavelength grating single slot waveguide; subwavelength grating double slot waveguide; racetrack ring resonator; refractive index sensor

\section{Introduction}

Silicon wire waveguides (WGs) are proficient in strong optical confinement into the narrow-area $(<500 \times 500 \mathrm{~nm})$ due to the high index contrast in the Silicon $(\mathrm{Si}) / \mathrm{Silica}\left(\mathrm{SiO}_{2}\right)$ system. This allows sharp bends with a radius as small as $5 \mu \mathrm{m}$ resulting in miniaturized photonic integrated circuits. Recently stated, a slot WG is a light guiding structure that can intensify the optical field in a nanoscale void (slot) of low refractive index material (can be air, $n=1.0$ ) inserted between higher refractive index material (rails) [1,2]. Considering a high index-contrast interface, Maxwell's equations suggest that the corresponding electric field must experience a large discontinuity with much higher amplitude in the low index region to comprehend the continuity of the normal part of electric flux density. A Si/ $/ \mathrm{SiO}_{2}$ platform is chemically secure and appropriate for on-chip gas [3,4] and bio-sensing [5] applications.

Subwavelength gratings (SWGs) can be used to craft an artificial media built on a microscopic scale to obtain the desired macroscopic behavior. A subwavelength grating for the silicon-on-insulator (SOI) platform can be created by the periodic arrangement of high index material (Si) and low index material $\left(\mathrm{SiO}_{2}\right)$ or other low index materials. Such periodic structures surmount diffraction and functions as a uniform medium provided that the periodicity does not follow Bragg's coupling condition criterion to other confined or radiative modes [6]. SWGs are commonly used as antireflective coatings [7], planar mirrors [8], broadband mirrors [9], fiber-chip couplers [10,11], modulators [12] and sensors $[13,14]$, among others. Light excites a Bloch mode in SWG WG, with a core comprised of a periodic arrangement of $\mathrm{Si}$ and $\mathrm{SiO}_{2}$ segments. In theory, this mode can propagate through the periodic WG segment with no losses incurred by diffraction into radiative or cladding modes. 
By manipulating the pitch, width and duty cycle of the SWG WG, the effective index of the medium can be locally engineered.

Several sensor designs based on SWG WGs are proposed in previous studies, which include ring resonators [15], grating [16] or photonic crystal [17]. Besides, there are other highly sensitive plasmonic sensor designs based on metal-insulator-metal WGs also presented [18-21]. The sensitivity of such devices is determined by the interaction between the electric field and the ambient medium, which can be improved by increasing the light-matter interaction. For conventional WGs, such as ridge or rib [22,23], due to the high index contrast of $\mathrm{Si}(\mathrm{n}=3.48 @ 1550 \mathrm{~nm})$ and $\mathrm{SiO}_{2}(\mathrm{n}=1.44 @ 1550 \mathrm{~nm})$, the utmost mode field power is confined in the WG core. One way to increase the sensitivity for TE polarized light is to decrease the core thickness of the WG which enhances the evanescent field that contributes to better light-matter interaction. A bulk sensitivity of $100 \mathrm{~nm} / \mathrm{RIU}$ is achieved [24], as demonstrated by TalebiFard et al., with $90 \mathrm{~nm}$ thick SOI strip WG.

In this paper, we analyzed four different configurations of slot WG, such as single slot waveguide (SSWG), double slot waveguide (DSWG), subwavelength grating single slot waveguide (GSSWG) and subwavelength grating double slot waveguide (GDSWG) using the finite element method (FEM). The modal characteristics of the WGs are studied in the first part of the paper and the dominance of SWG WGs on the standard slot WG schemes is manifested. In the second part of the paper, racetrack ring resonators based on the above mentioned WG schemes are studied, revealing an extraordinary sensing capability of the sensor utilizing SWG WGs, which outclasses the previously reported works.

\section{SWG Slot WG Geometry and Theory}

Bloch-Floquet formalism shows how electromagnetic waves are propagated in periodic media [6]. Based on the wavelength, propagation can be categorized into three wavelength regions for a specified grating period $(\Lambda)$ : (i) The sub-wavelength region in which the wavelength to period ratio is $\lambda / \Lambda>2 \cdot n_{\text {eff. }}$ This correlates to the wavelength range greater than the Bragg wavelength and the WG behaves as a standard WG. The periodic structure, in this case, retains a true lossless mode [25]. (ii) The wavelength spectrum is analogous to the photonic bandgap in which Bragg reflections take place. (iii) The wavelength range shorter than the Bragg wavelength, where the Bloch wave is leaky and part of the energy, is radiated out of the WG and the propagation loss is determined by reflection and diffraction at the segment boundaries caused by the high refractive index difference between air and silicon.

By having $\Lambda<<\lambda$, the mode is lossless since the reflection and diffraction effects are concealed. This is analogous to the distribution of electrons in periodic potentials, as in semiconductor materials. SWG WGs are attractive because they allow tailored propagation properties by varying the period $\left(l_{\text {grat }}+d\right)$, WG width $\left(W_{\text {rail }}\right)$ and WG height $\left(H_{\text {rail }}\right)$. Our suggested SWG WG scheme is based on slot WG, which is divided into periodic slot segments with linewidth $\left(l_{\text {grat }}\right)$. A slot WG has recently been implemented as a novel WG structure to confine and direct light in a nanometer-sized low refractive index material. A slot WG consists of two strips (rails) of a high-index material separated by a narrow low-index (slot) region. Thanks to its outstanding features, the slot WG is highly attractive for sensing applications [26].

In the case of a typical slot WG, the light is confined at the interface between highindex-contrast materials in the $x y$-plane by the electric field discontinuity, and high optical intensity can be obtained in the slot. The periodicity in the z-direction $\left(n^{2}(z)=n^{2}\left(z+l_{\text {grat }}+d\right)\right)$ guarantees that the wave vector $k_{z}$ is still preserved. An electromagnetic solution takes the following form, according to the Bloch (or Floquet) theorem:

$$
E=E_{K}(x, y, z) e^{-i K z}
$$

where $K$ is the Bloch wavenumber and $E_{K}(x, y, z)$ is a periodic function with period $\left(l_{g r a t}+d\right)$, so that $E_{K}(x, y, z)=E_{K}\left(x, y, z+l_{\text {grat }}+d\right)$.Similar to the dispersion relationship for typical WGs, the dispersion relation for SWG WGs is $\omega=\omega(K)$. Depending on the spectral regime, the Bloch wave vector $K$ can either be real or complex. If $K$ is real, the Bloch wave intensities will be a periodic function of position 
in the medium and propagate with no loss. There are analytical solutions for a layered structure with uniform material properties in the $x y$-plane, but not for the case of index driven modes (vertical and lateral confinement) and computational techniques should be used.

Figure 1 represents the schematic of an SSWG, DSWG, GSSWG and GDSWG. The structure is designed on a silicon-on-insulator (SOI) platform with a $220 \mathrm{~nm}$ thick top silicon layer (h) and a $3 \mathrm{um}$ thick buried oxide (BOX) layer. The geometric parameters of the WGs are tabulated in Table 1.

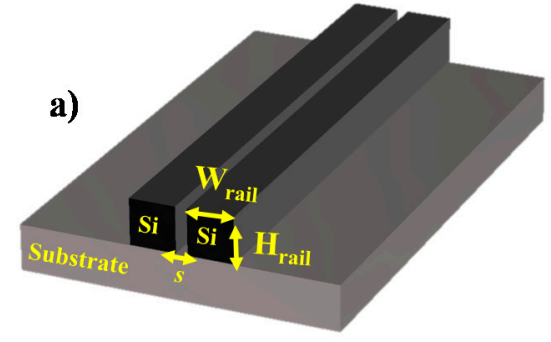

b)

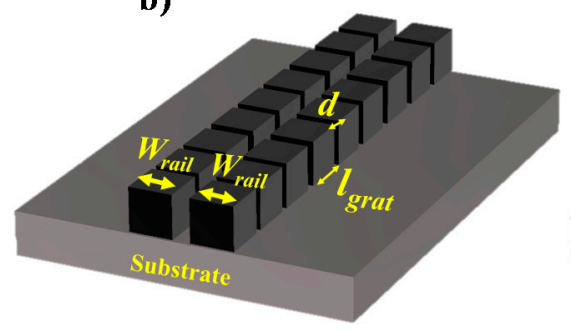

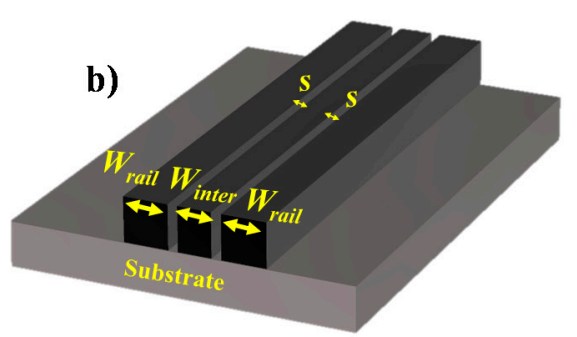

d)

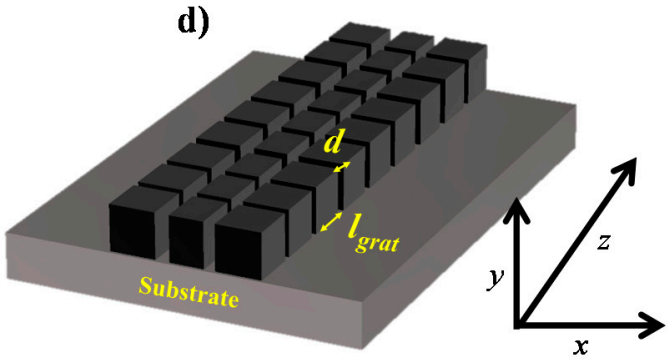

Figure 1. Schematic of (a) Single slot waveguide, (b) Double slot waveguide (c) Subwavelength grating single slot waveguide, (d) Subwavelength grating double slot waveguide.

Table 1. Geometric parameters of WGs.

\begin{tabular}{ccccccc}
\hline $\begin{array}{c}\text { For All Four } \\
\text { WG } \\
\text { Schemes }\end{array}$ & $\begin{array}{c}\text { SSWG } \\
\text { and } \\
\text { GSSWG }\end{array}$ & $\begin{array}{c}\text { For All Four } \\
\text { WG } \\
\text { Schemes }\end{array}$ & $\begin{array}{c}\text { DSWG } \\
\text { and } \\
\text { GDSWG }\end{array}$ & $\begin{array}{c}\text { GSSWG } \\
\text { and } \\
\text { GDSWG }\end{array}$ & $\begin{array}{c}\text { GSSWG } \\
\text { and } \\
\text { GDSWG }\end{array}$ & $\begin{array}{c}\text { DSWG } \\
\text { and } \\
\text { GDSWG }\end{array}$ \\
\hline$H_{\text {rail }}(\mathrm{nm})$ & $W_{\text {rail }}(\mathrm{nm})$ & $s(\mathrm{~nm})$ & $W_{\text {rail }}(\mathrm{nm})$ & $l_{\text {grat }}(\mathrm{nm})$ & $d(\mathrm{~nm})$ & $W_{\text {inter }}(\mathrm{nm})$ \\
\hline 220 & $200-400$ & 50 & $200-400$ & 250 & $50-100$ & 150 \\
\hline
\end{tabular}

The WG models are simulated using the 3D finite element method (FEM) based model in COMSOL Multiphysics 5.1. The E-M wave frequency domain (emw) was used as a physics interface. In COMSOL simulations, the sub-domains in the WG cross-section are divided into triangular mesh elements with a "very fine" mesh grid size for the entire WG design. The meshing relies on the precision of the solution and the computational capacity of the system used. The meshing used in this work provides precise simulation results based on our system processing speed. For wave propagation systems, a domain with open computational domain boundaries is necessary as it allows the EM wave to travel without any reflection. The open geometry is determined by assigning scattering boundary conditions (SBC) at the outer edges of the simulation window.

The transmission spectrum of GDSWG with $\Lambda=300 \mathrm{~nm}$ (Duty cycle $(\eta)=0.833$ ) is plotted over a wavelength range of 1000-3000 nm, as shown in Figure 2. The transmission spectrum is determined using the following expression: Transmission $(d B)=10 \times \log \frac{P_{\text {out }}}{P_{\text {in }}}$, where $P_{\text {out }}$ and $P_{\text {in }}$ are the output power and input power, respectively. The Bloch mode propagates in the direction normal to the periodic structures with a wavevector $\mathrm{k}=2 \pi / \lambda$ and a temporal frequency $\omega$. The resonant region where $\Lambda \sim \lambda / 2$ and where the wave is not transmitted is referred to as a photonic bandgap. However, in the subwavelength regime $\Lambda<\lambda / 2$, the structure behaves as a homogeneous effective medium with an effective index $n_{\text {eff }}=c(k / \omega)$, where $\mathrm{c}$ is the velocity of light. The mode is lossless since it conceals the 
reflection and diffraction effects. The wavelength region between $\sim 1070-1220 \mathrm{~nm}$ is photonic bandgap for the designed WG, whereas the region over $\sim 1240 \mathrm{~nm}$ is the subwavelength region which will be used as an operational wavelength for the ring resonators presented in Section 4.

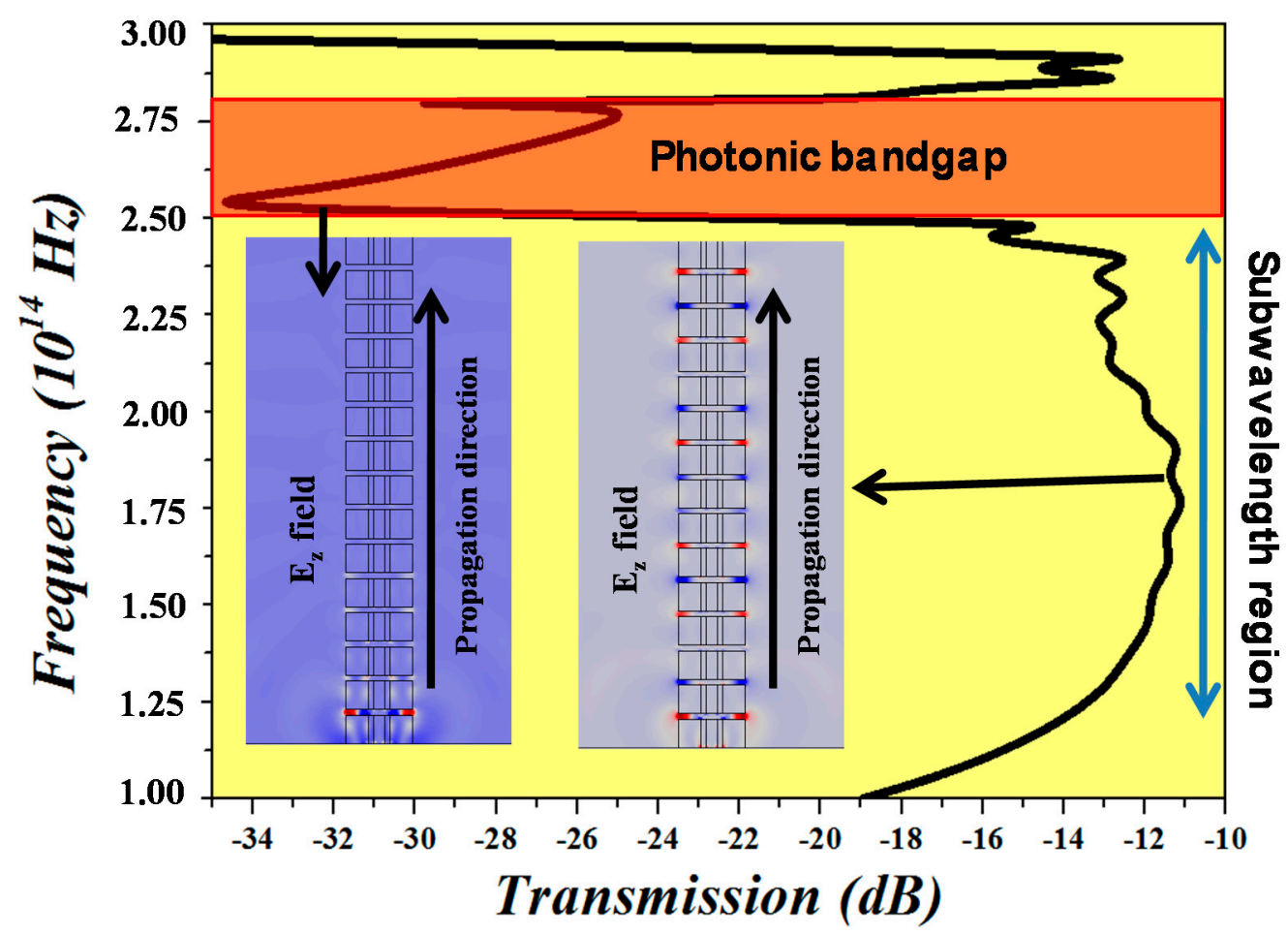

Figure 2. The transmission spectrum of GDSWG which is divided into two regions-photonic bandgap and subwavelength region. Inset of the figure shows the $E_{z}$ plot of a WG in both the regions.

\section{Mode Sensitivity Analysis of Single and Double Slot WG}

In this section, the mode sensitivity ( $S_{\text {mode }}$ ) analysis of SSWG and DSWG is performed, which reveals the dominance of double slot WG in terms of light-matter interaction. The effective refractive index $\left(n_{\text {eff }}\right)$ of SSWG and DSWG is calculated at $\lambda=1550 \mathrm{~nm}$ by varying the $W_{\text {rail }}$ in the range of 200 to $400 \mathrm{~nm}$. The remaining parameters, such as s and $W_{\text {inter }}$ (for DSWG), are fixed at 50 and $150 \mathrm{~nm}$, respectively. The real part of $n_{\text {eff }}$ of SSWG and DSWG at $\mathrm{n}=1.0$ and 1.35 is plotted in Figure $3 a$, which increases as $W_{\text {rail }}$ increases and allows the formation of dielectric mode in the rail. $S_{\text {mode }}$ is calculated using the following formula:

$$
S_{\text {mode }}=\frac{\Delta n_{\text {eff }}}{\Delta n}
$$

where $\Delta n_{\text {eff }}$ is the change in effective refractive index due to change in the refractive index of the ambient medium $(\Delta \mathrm{n})$. From Figure $3 \mathrm{~b}$, it can be seen that $S_{\text {mode }}$ of both SSWG and DSWG is strictly dependent on $W_{\text {rail }}$, which decreases as $W_{\text {rail }}$ increases. At $W_{\text {rail }}=230 \mathrm{~nm}$, the maximum $S_{\text {mode }}$ of 0.8 and 0.831 for SSWG and DSWG is obtained, which is due to the maximum overlap of the evanescent field tail in the slot region, respectively. However, at $\mathrm{W}_{\text {rail }}=400 \mathrm{~nm}$, the high index cladding region is large enough to support dielectric mode. As a result, the mode confined in the low index slot region splits up and the mode power in the slot is reduced, which brings to the $S_{\text {mode }}$ of both WGs to $\sim 0.34$. The E-field distribution of the mode in SSWG and DSWG at $W_{\text {rail }}=200$ and $400 \mathrm{~nm}$ is shown in Figure 3c. 

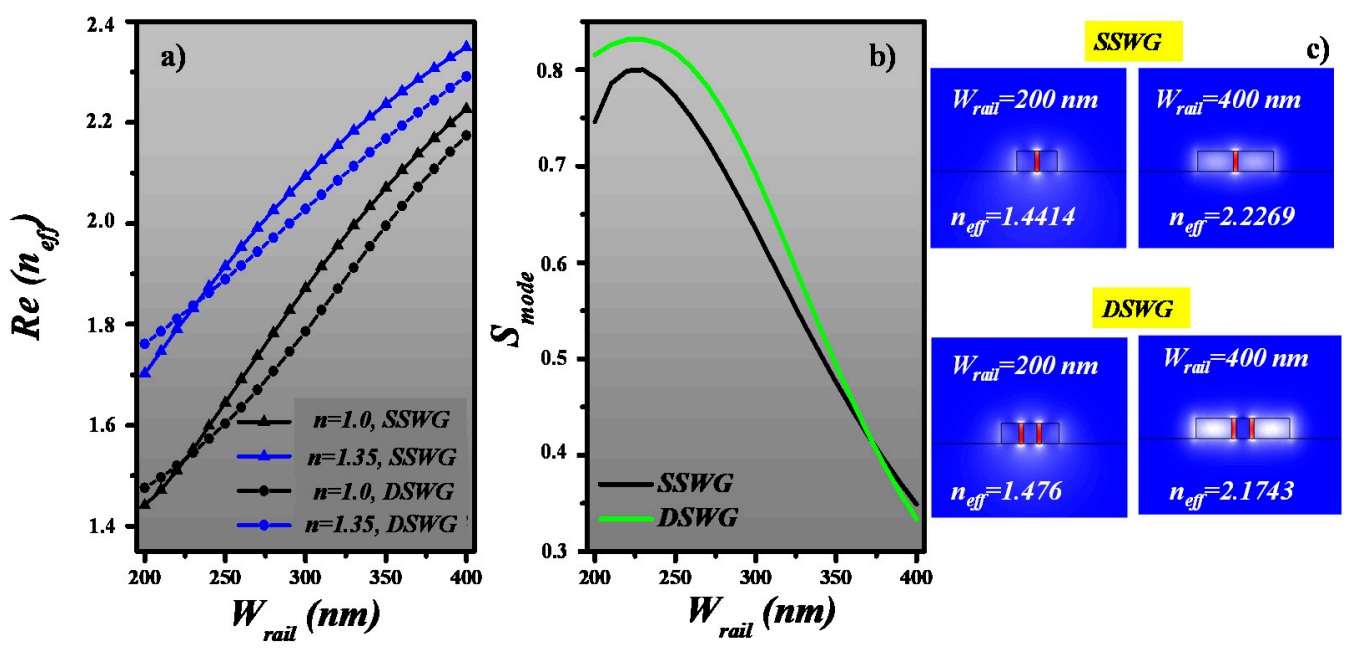

Figure 3. (a) Real part of the effective refractive index of SSWG and DSWG, (b) Mode sensitivity analysis, (c) E-field distribution in SSWG and DSWG at $W_{\text {rail }}=200$ and $400 \mathrm{~nm}$.

From $S_{\text {mode }}$ analysis, it is evident that double slot WG configuration is more sensitive than single slot WG. Along with the low index slot region, SWG WGs has air gaps between silicon segments along the propagation direction of light. The propagating mode power is confined in the slot as well as in the gaps between the grating segments. This combined power exposed to the ambient medium can result in an elevated $S_{\text {mode }}$, which is proven in Section 4 . The E-field distribution of the TE-polarized light in SSWG, DSWG, GSSWG and GDSWG is calculated at $\lambda=1550 \mathrm{~nm}$, which lies in the subwavelength region. The geometric parameters such as $\mathrm{H}_{\text {rail }}, \mathrm{W}_{\text {rail }}, \mathrm{s}, \mathrm{W}_{\text {inter }}$ are fixed at 220, 200, 50 and $150 \mathrm{~nm}$, respectively. The period of SWG WGs is chosen to be $300 \mathrm{~nm}$ with $\eta=0.833$. The cross-sectional view (first column), top view (second column) and line graph (third column) of the WGs with E-field distribution are displayed in Figure 4. The cross-section view is taken in the middle of the WG (for SWG WGs, it is taken in the middle of the silicon grating segment i.e., $\left.l_{\text {grat }} / 2\right)$, whereas the top view is taken at $H_{\text {rail }} / 2$. The linecut profile of electric field intensity $\left(|\mathrm{E}|^{2}\right)$ is taken in the middle of the respective WGs. There is no evident difference in the E-field distribution of the propagating mode in the standard slot WGs (Figure 4a,b) and SWG slot WGs (Figure 4c,d).

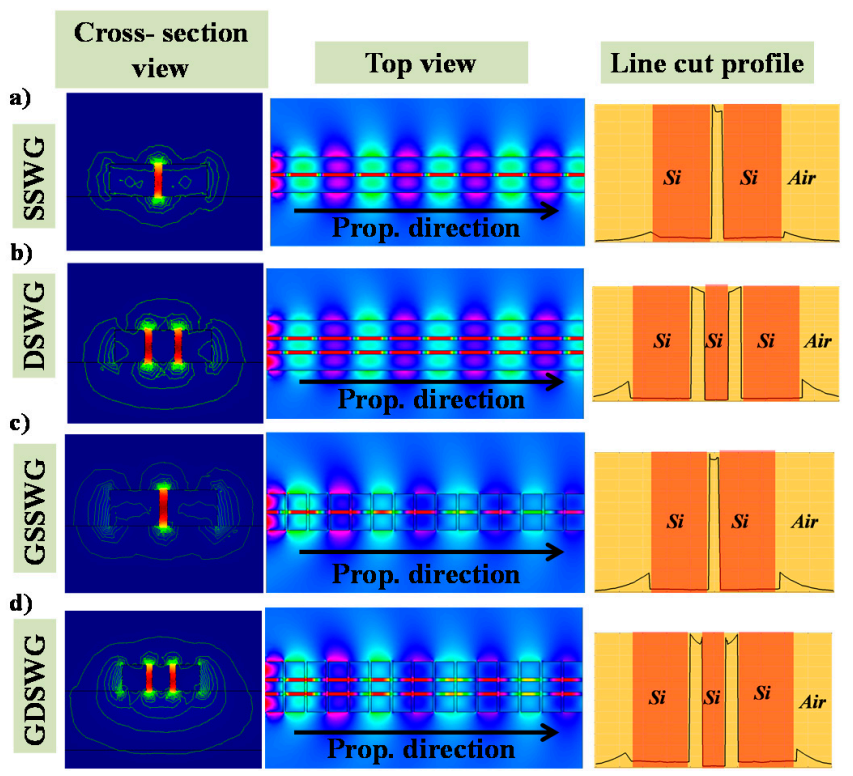

Figure 4. E-field distribution in the cross-sectional view, top view and line cut profile of electric field intensity of (a) SSWG, (b) DSWG, (c) GSSWG, (d)GDSWG. 
The WG structures are optimized at $\lambda=1550 \mathrm{~nm}$ to obtain a single-mode operation with high sensitivity to the waveguide cladding, while meeting the minimum feature size limitations imposed by the fabrication constraints ( $\sim 40 \mathrm{~nm}$ gap size using electron-beam lithography and reactive-ion etching). The mode sensitivity can be directly derived from the dispersion diagrams corresponding to different cladding refractive index values. It is worth noting that the sensitivity of mode can be related to the overlap integral in the cladding $\left(\Gamma_{c}\right)$ [15], which is the sum of the overlap factors of the following three regions:

1. For SSWG and DSWG, the slot region/s between silicon grating segment with a volume of $s \times W G_{\text {length }} \times H_{\text {rail }}$ and $2 \times s \times W G_{\text {length }} \times H_{\text {rail }}$, respectively, whereas for GSSWG and GDSWG, the slot region/s between two silicon segments $\left(\Gamma_{\text {slot }}\right.$ and $\left.\Gamma_{\text {slots }}\right)$ with a volume of $s \times l_{\text {grat }} \times H_{\text {rail }}$ and $2 \times\left(\mathrm{s} \times l_{\text {grat }} \times H_{\text {rail }}\right)$, respectively.

2. For GSSWG, the gap region between the periodic silicon segments $\left(\Gamma_{\text {gap }}\right)$ with a volume of $\left(2 \times \mathrm{W}_{\text {rail }}+\mathrm{s}\right) \times d \times \mathrm{H}_{\text {rail }}$. In the case of GDSWG, it is calculated as $\left(2 \times \mathrm{W}_{\text {rail }}+\mathrm{W}_{\text {inter }}+2 \times \mathrm{s}\right) \times$ $\mathrm{b} \times H_{\text {rail }}$.

3. The remaining upper cladding medium $\left(\Gamma_{u c}\right)$.

For the SWG WGs, the electric field intensity varies periodically along the propagation direction. Consequently, $\Gamma_{\text {subregion }}$ can be calculated by integrating the intensity over the volume of subregion (slot, gap, upper cladding) in a single unit cell volume with a period $(\Lambda)$ by using the following formula:

$$
\Gamma_{\text {subregion }}=\frac{\iiint_{\text {subregion }}|\mathrm{E}(\mathrm{x}, \mathrm{y}, \mathrm{z})|^{2} \mathrm{dxdydz}}{\iiint_{\text {unit cell }}|\mathrm{E}(\mathrm{x}, \mathrm{y}, \mathrm{z})|^{2} \mathrm{dxdydz}}
$$

where $\Gamma_{\mathrm{c}}=\Gamma_{\text {slot }}+\Gamma_{\text {gap }}+\Gamma_{\text {uc. }}$

In Figure $5 a$, the mode power confinement in all the four WG schemes is presented. For SSWG and DSWG, the mode confinement is the intensity integration in one slot for SSWG or two slots for DSWG. However, in the case of GSSWG and GDSWG, the mode power in the gaps between silicon grating segments is also considered, as mentioned above. It can be seen that SSWG and DSWG possess maximum mode confinement at $W_{\text {rail }}=200 \mathrm{~nm}$, which falls rapidly as $W_{\text {rail }}$ increases due to the formation of dielectric mode in the silicon rail. This suggests that the performance of these WGs are strictly dependent on their dimension. Small fabrication error can reduce the mode power, which results in the low overlap integral, as shown in Figure 5b. However, these WGs have relatively low transmission loss $(\sim 3 \mathrm{~dB}$ to $10 \mathrm{~dB})$ as compared to SWG WGs, which is calculated using the expression: $10 \times \log \left(P_{\text {out }} / P_{\text {in }}\right)$, as shown in Figure $5 c$.
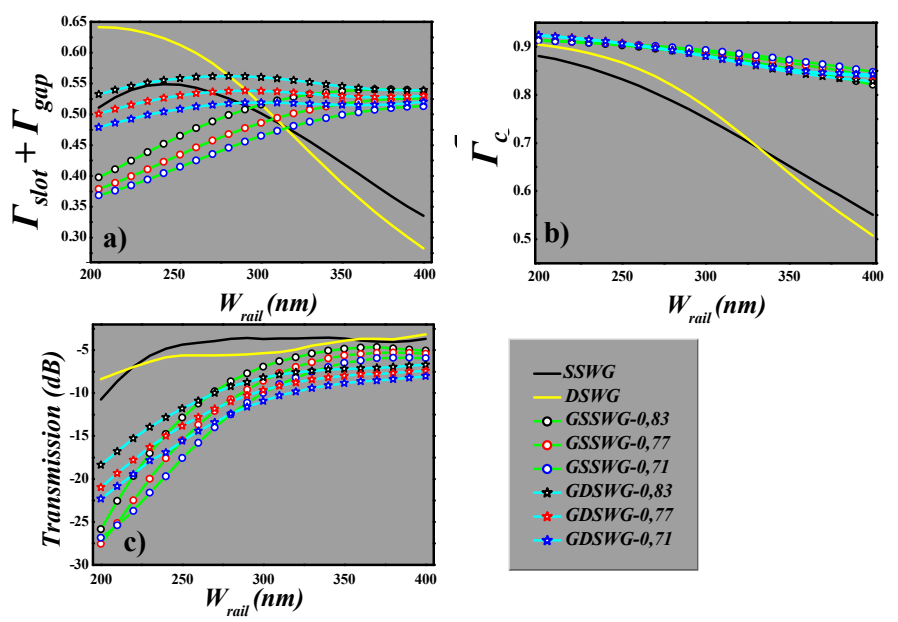

Figure 5. Variation of (a) $\Gamma_{\text {slot }}+\Gamma_{\text {gap }},(\mathbf{b}) \Gamma_{\mathcal{C}},(\mathbf{c})$ Transmission $(\mathrm{dB})$, on the WG width $\left(W_{\text {rail }}\right)$. 
On the other hand, three different duty cycles $(0.83,0.77$ and 0.71$)$ are selected for GSSWG and GDSWG to calculate the mode confinement, overlap integral and transmission versus $W_{\text {rail }}$ in the range of 200 to $400 \mathrm{~nm}$. It can be seen that mode confinement and overlap integral is least affected by variation in $W_{\text {rail }}$. The maximum mode confinement and overlap integral of 0.56 and 0.92 are obtained when $W_{\text {rail }}$ is in the range of 200 to $250 \mathrm{~nm}$, respectively. The transmission loss of SWG WGs is relatively high compared to standard slot WGs. However, these losses can be reduced by increasing $\mathrm{W}_{\text {rail }}$ from 200 to $400 \mathrm{~nm}$. The transmission loss of $\sim 6 \mathrm{~dB}$ can be obtained at $\mathrm{W}_{\text {rail }}=400 \mathrm{~nm}$, however, at the cost of low overlap integral which leads to lower sensitivity.

\section{Towards Highly Sensitive SWG Racetrack Ring Resonator Design}

Refractive index sensors demonstrate several applications in the biological and chemical fields and have been widely studied in recent years, such as solution concentration and $\mathrm{pH}$ value, which can be estimated based on refractive index change. The measurement of changes in resonance wavelength is the most common interrogation method in ring resonators. In this section, we studied four configurations of racetrack ring resonators based on different slot WG schemes such as SSWG, DSWG, GSSWG and GDSWG, as shown in Figure 6. In all the four cases, the ring resonator is side coupled to a standard strip WG. In Figure 6a,b, ring resonator designs based on SSWG and DSWG are presented, respectively, whereas in Figure 6c,d, GSSWG and GDSWG race track ring resonators are presented. The period $(\Lambda)$ of the grating $W G$ is fixed at $300 \mathrm{~nm}$ with $\eta=0.83$. For a fair analysis, the coupling length $\left(c_{l}\right)$ of all four ring resonator designs is fixed to $3000 \mathrm{~nm}$. For a better understanding of the device design, the geometric parameters are stated in Table 2.
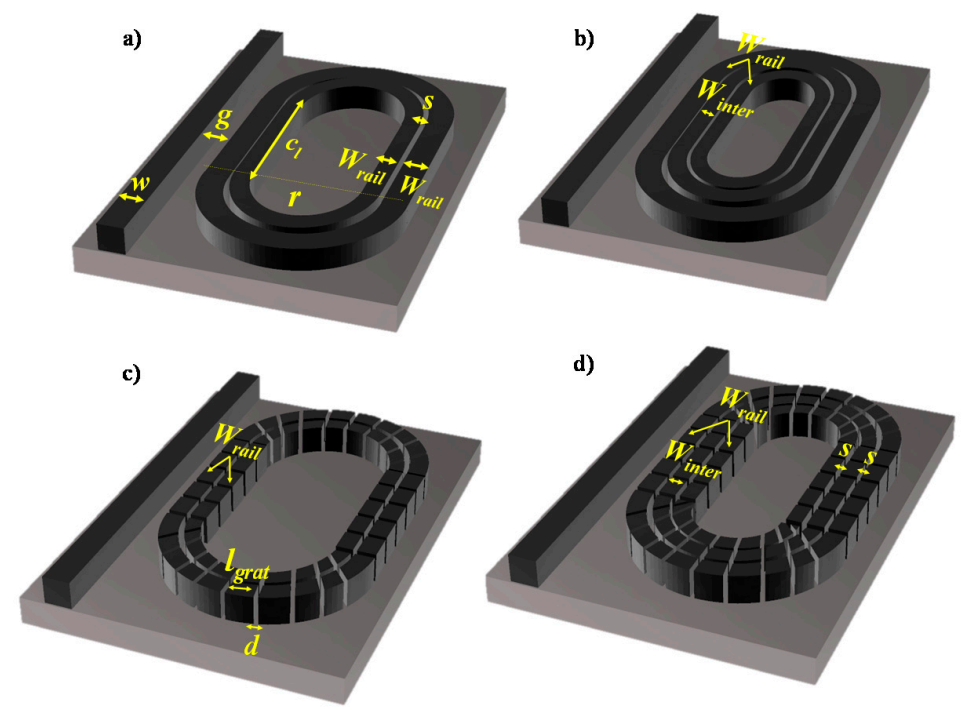

Figure 6. Schematic of race track resonator based on (a) SSWG, (b) DSWG, (c) GSSWG, (d) GDSWG.

Table 2. Race track resonator parameters.

\begin{tabular}{lcccccccccc}
\hline $\begin{array}{c}\text { WG } \\
\text { Type }\end{array}$ & $\begin{array}{c}\boldsymbol{w} \\
(\mathbf{n m})\end{array}$ & $\boldsymbol{g} \mathbf{( \mathbf { n m } )}$ & $\begin{array}{c}W_{\text {rail }} \\
(\mathbf{n m})\end{array}$ & $\begin{array}{c}s \\
(\mathbf{n m})\end{array}$ & $\begin{array}{c}\text { Slot } \\
\text { Displacement } \\
(\mathbf{n m})\end{array}$ & $\begin{array}{c}W_{\text {inter }} \\
(\mathbf{n m})\end{array}$ & $\begin{array}{c}\boldsymbol{c}_{l} \\
(\mathbf{n m})\end{array}$ & $\begin{array}{c}\boldsymbol{l}_{\text {grat }} \\
(\mathbf{n m})\end{array}$ & $\begin{array}{c}d \\
(\mathbf{n m})\end{array}$ & $\begin{array}{c}r \\
(\mathbf{n m})\end{array}$ \\
\hline SSWG & 400 & 100 & 200 & 50 & $0-100$ & - & 3000 & - & - & 5000 \\
DSWG & 400 & 100 & 200 & 50 & $0-100$ & 150 & 3000 & - & - & 5000 \\
GSSWG & 400 & 100 & 200 & 50 & $0-100$ & - & 3000 & 250 & 50 & 5000 \\
GDSWG & 400 & 100 & 200 & 50 & $0-100$ & 150 & 3000 & 250 & 50 & 5000 \\
\hline
\end{tabular}

From the E-field distribution profiles presented in Figure 4, it is quite evident that in contrast to the evanescent field on the top surface and sidewalls of the WG, there is a considerably stronger mode field existing on the light propagation path between silicon grating segments. This gives SWG-based 
microring biosensors an extended surface sensing region on the propagation path, and thus, a distinctive advantage in surface sensing over microrings based on standard WGs. Therefore, surface sensitivity is an important figure of merit. In a resonance-based sensing method, surface sensitivity $S_{S}$ can be defined as the resonance wavelength shift per the change of surface layer thickness [27].

$$
S_{s}=\frac{\Delta \lambda}{\Delta t}=\frac{\lambda}{n_{g}}\left(\frac{\partial n_{e f f}}{\partial t}\right)
$$

where $n_{g}$ is group index and $t$ is the thickness of the surface layer. The detailed analysis of surface sensitivity can be found in reference [15]. However, in this work, we have studied the bulk sensitivity of the ring resonators based on the WG schemes studied in this paper.

Before analyzing the sensing capability of the proposed sensor design, the resonance condition and extinction ratio $(E R)$ is determined by displacing the slot towards the inner periphery of the ring. ER is calculated using the following expression:

$$
E R=10 \times \log \frac{P_{\text {out }}}{P_{\text {in }}},
$$

where $P_{\text {out }}$ and $P_{\text {in }}$ are the output and input power at $\lambda_{\text {res }}$, respectively. The $W_{\text {rail }}, s$ and $g$ are maintained at 200, 50 and $100 \mathrm{~nm}$, respectively. The slot is displaced towards the inner periphery of the ring with a step size of $10 \mathrm{~nm}$, which helps to find the optimal position where the maximum mode confinement

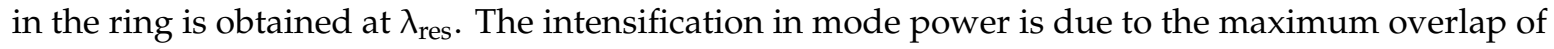
the evanescent tail of the propagating mode in the slot. The resonance wavelength and ER of SSWG and DSWG based ring resonator designs are plotted in Figure $7 \mathrm{a}, \mathrm{b}$, respectively. The $\lambda_{\text {res }}$ performs a blueshift with slot displacement increases from 0 to $\sim 30 \mathrm{~nm}$. However, slot displacement greater than $\sim 40 \mathrm{~nm}$ results in a redshift of $\lambda_{\text {res }}$. The ER has a significant impact on the placement of the slot, which is evident in Figure $7 \mathrm{~b}$. The ER of $<10 \mathrm{~dB}$ is obtained for both symmetric SSWG and DSWG based ring resonator design. However, an optimized slot displacement of $60 \mathrm{~nm}$ results in a high ER of 22 and $31.2 \mathrm{~dB}$ for SSWG and DSWG based ring resonators, respectively.
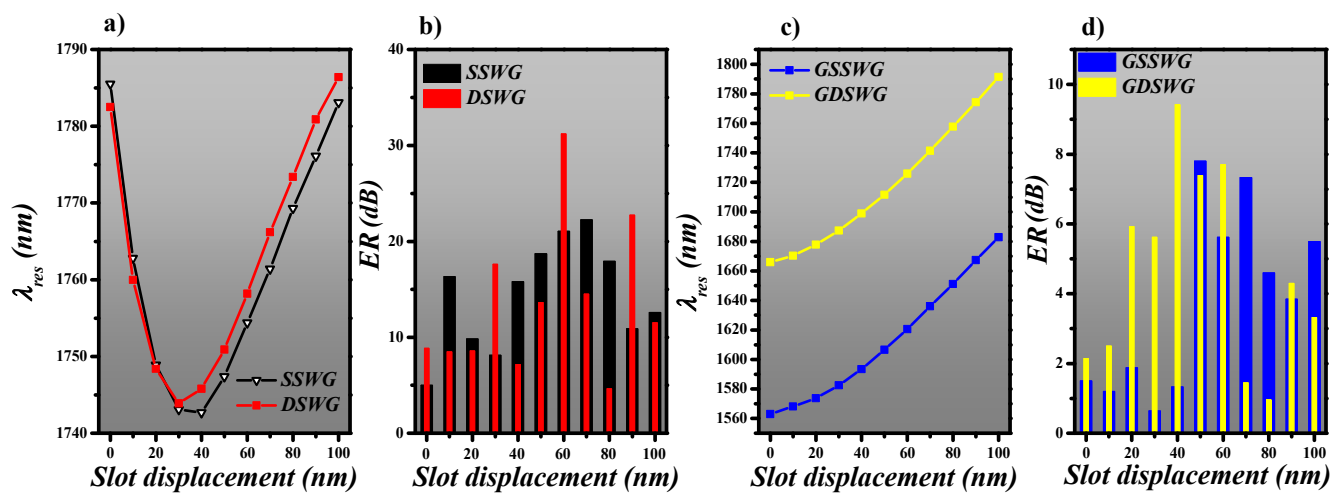

Figure 7. Determination of resonance wavelength of (a) SSWG and DSWG, (b) GSSWG and GDSWG. Extinction ratio (ER) of (c) SSWG and DSWG, (d) GSSWG and GDSWG.

Figure $7 \mathrm{c}, \mathrm{d}$ present the $\lambda_{\text {res }}$ and ER of GSSWG and GDSWG based ring resonator designs, respectively. The $\lambda_{\text {res }}$ performs a redshift with an increasing slot displacement towards the inner periphery of the ring, as shown in Figure 7c. As these WGs are refractive index modified and silicon grating segments are periodically arranged along the propagation direction of the light, that is why the mode coupling at $\lambda_{\text {res }}$ is weaker than a standard WG design. This reduces the ER of the ring resonators based on SWG WGs, but is still high enough to interpret the resonance dips in the spectrum. The maximum ER of 7.8 and $9.4 \mathrm{~dB}$ is obtained for GSSWG and GDSWG, respectively, as shown in Figure 7d. 


\section{Sensor Performance}

The optical resonances are acquired by filling the medium with a material of $\mathrm{n}=1.0002-1.0005$, which is equivalent to the refractive index of several toxic gases, such as $\mathrm{CO}_{2}, \mathrm{CH}_{4}$ and $\mathrm{CO}$, etc. The most rigorous method is a full 3D FEM approach. Nevertheless, for large structures, this approach is computationally very exhausting and for that reason, not appropriate for large parameter sweeps. Therefore, the transmission spectrum and E-field distribution are simulated using 2D-FEM. Mode sensitivity analysis of SSWG, DSWG, GSSWG and GDSWG presented in the previous section suggests that ring resonators based on SWG WGs can boost the mode power in the upper cladding. Therefore, elevated sensitivity can be expected. High sensitivity is always attractive in these sensors, which strongly depends on light polarization, optical loss and the light-matter interaction. Sensitivity is calculated by using the following expression:

$$
S=\Delta \lambda / \Delta n
$$

where $\Delta \lambda$ represents the shift of the sensor resonance in $\mathrm{nm}$ and $\Delta n$ is the difference of the RI in the medium. The microring resonators based on SWG WGs were first demonstrated with bulk sensitivity $\left(\Delta \lambda_{\text {res }} / \Delta n\right)$ of $400-500 \mathrm{~nm} / \mathrm{RIU}$ [28], which is several times higher than conventional microring resonators based on strip WGs. The figure of merit $(F O M)$ is another parameter which should also be considered while designing the ring resonator sensor. FOM is expressed as $S / F W H M$, where $F W H M$ is full width at half maximum. Chrostowski et al. [29] suggested the intrinsic limit of detection (iLOD) as a figure of merit independent on readout circuitry and data processing, which is expressed as $i L O D=\frac{\lambda_{\text {res }}}{S \times Q-f a c t o r}$ and reflects the detection capabilities of change in refractive index. The $Q-f a c t o r$ is defined as $\lambda_{\text {res }} / F W H M$. Integrated resonators with high $Q$-factors are particularly advantageous for a wide range of applications such as narrow band width filters, high performance lasers, high-efficiency non-linear optic devices and high sensitivity sensors.

The E-field distribution in SSWG, DSWG, GSSWG and GDSWG based racetrack ring resonators is shown in Figure 8a-d. For each design, the optimal slot displacement value is selected, where the maximum $E R$ is obtained as labeled in Figure 8. It can be seen in Figure $8 \mathrm{c}$,d, the E-field is prominently enhanced in the grating segment, which provides a strong light-matter interaction. The ring resonators are highly responsive to the ambient refractive index. A slight change in the refractive index can lead to a significant shift in the resonance wavelength $\left(\lambda_{\text {res }}\right)$.

a)
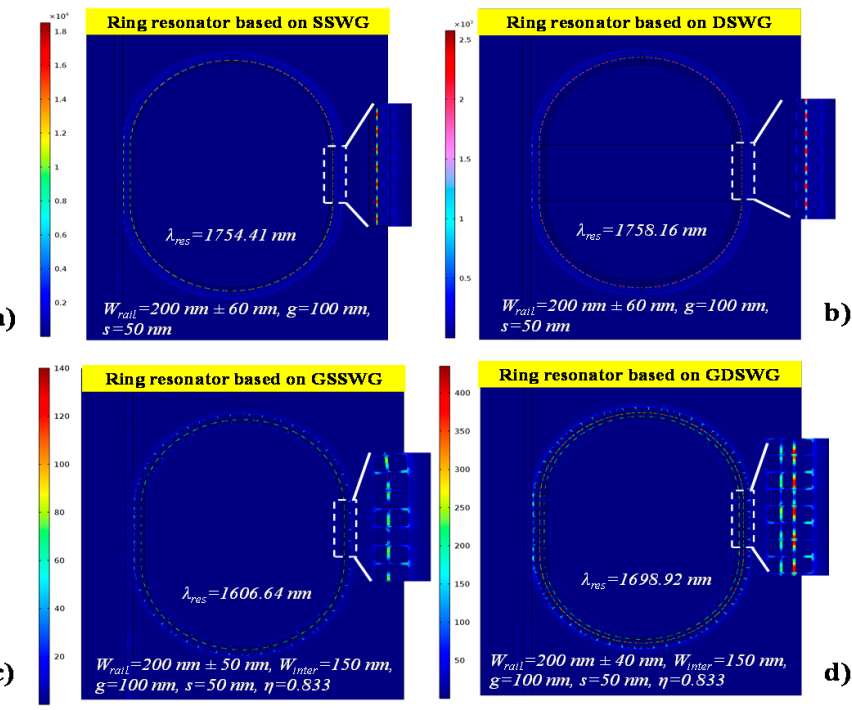

Figure 8. E-field distribution in (a) SSWG resonator, (b) DSWG resonator, (c) GSSWG resonator, (d)GDSWG resonator. The inset shows the zoomed section of the ring resonator at $\lambda_{\text {res }}$. 
The S, FOM and Q-factorof all the designs are calculated and displayed in Figure 9. It can be seen from Figure 9a that the GDSWG based resonator has almost 2.5 times higher sensitivity than the resonator design based on the standard SSWG. The narrow FWHM of the DSWG ring resonator obtained at $\lambda_{\text {res }}$ is $\sim 0.04 \mathrm{~nm}$ (at optimized WG geometry). Therefore, the FOM of the ring resonator design based on DSWG is 12,270 , which is higher than the remaining three sensor designs, as shown in Figure 9b). Q-factor helps quantify the losses in the resonator. The sensor designs based on SSWG and DSWG show a high Q-factor of 10,668 and 43,150 at optimized WG parameters, respectively. The iLOD of SSWG, DSWG, GSSWG and GDSWG based sensor designs are $4.33 \times 10^{-4} \mathrm{RIU}, 8.15 \times 10^{-5} \mathrm{RIU}$, $1.05 \times 10^{-3} \mathrm{RIU}, 3.12 \times 10^{-4} \mathrm{RIU}$, respectively.
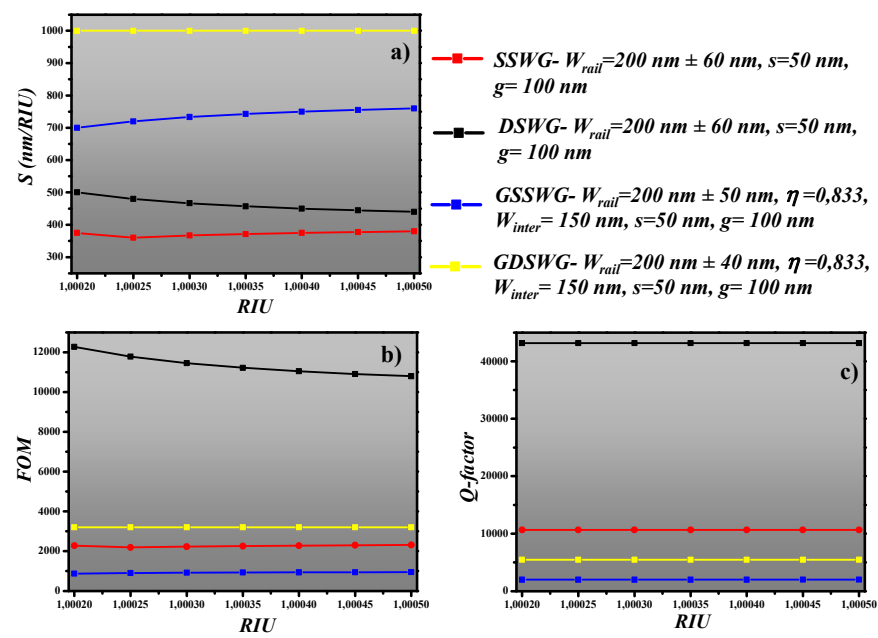

Figure 9. (a) Sensitivity, (b) FOM, (c) Q-factor of all four designs.

In Table 3, we have listed several recently proposed and demonstrated refractive index sensors based on an SOI platform. However, our main proposed designs based on GSSWG and GDSWG outclass all the previous reports [5,24,28,30-37] with an exceptionally high sensitivity of 760 and $1000 \mathrm{~nm} / \mathrm{RIU}$ (at optimized parameters), respectively. The parameters used in our study are practical and achievable with existing standard CMOS fabrication technology.

Table 3. Previously reported sensitivities of SOI ring resonators.

\begin{tabular}{cccc}
\hline No. & Resonator Designs & Sensitivity (nm/RIU) & Reference \\
\hline 1 & Strip WG ring resonator & 100 & 24 \\
\hline 2 & SWG strip WG ring resonator & $400-500$ & 28 \\
\hline 3 & Bragg grating slot WG & 340 & 30 \\
\hline 4 & Strip WG ring resonator & 439 & 31 \\
\hline 5 & Slot WG ring resonator & 212.1 & 32 \\
\hline 6 & Single semiconductor nanowire & 235 & 33 \\
\hline 7 & Wire WG ring resonator & 135 & 34 \\
\hline 8 & Strip WG ring resonator & 270 & 35 \\
\hline 9 & Silicon microring & 222 & 37 \\
\hline 10 & SWG double slot microring & 840 & 5 \\
\hline 11 & Slot WG ring resonator & 298 & This work \\
\hline 12 & SSWG racetrack ring resonator & 380 & This work \\
\hline 13 & DSWG racetrack ring resonator & 500 & This work \\
\hline 14 & GSSWG racetrack ring resonator & $700-760$ & This work \\
\hline 15 & GDSWG racetrack ring resonator & 1000 &
\end{tabular}




\section{Concluding Remarks}

In this paper, a novel design of a racetrack ring resonator composed of the subwavelength grating double slot waveguide is proposed for gas sensing application. Different configurations of slot waveguides, such as single slot waveguide, double slot waveguide, subwavelength grating single and double slot waveguides, are studied and compared via the finite element method. Subwavelength grating slot waveguides are a special type of optical waveguides, where quasi-TE mode experiences high disruption at the interface between the low index region (slot) and gaps between the silicon segment result in high electric field intensity. This characteristic makes subwavelength grating waveguides a promising candidate for applications that involve strong light-matter interaction, such as sensing and non-linear photonics. The sensing capability of the device can be significantly enhanced, which is not possible to attain with conventional slot waveguides. Our proposed design is capable of providing a sensitivity of $1000 \mathrm{~nm} / \mathrm{RIU}$, which is approximately $2.5 \times$ higher than the values that can be obtained via standard slot waveguide ring resonators of the same geometric parameters. The transmission loss of subwavelength grating waveguides can be significantly high if not designed properly. Therefore, there is always a compromise between sensitivity and $Q$-factor. The waveguide dimensions at which the sensor device is highly sensitive can offer a low $Q$-factor $~ 5445$. The FOM and Q-factor of such devices can be improved by increasing the width of the waveguide at the cost of reduced sensitivity. On the other hand, the ring resonator based on DSWG offers a maximum $Q$-factor and limit of detection of 43,150 and $8.15 \times 10^{-5}$ RIU, respectively.

Author Contributions: We declare an equal contribution of all the authors to finalize this paper in its presentable form. All authors have read and agreed to the published version of the manuscript.

Funding: This work was financially supported by the Ministry of Science and Higher Education within the State assignment FSRC «Crystallography and Photonics» RAS (No. 007-GZ/Ch3363/26) for numerical calculations and Russian Science Foundation (No. 20-69-47110) for theoretical results.

Acknowledgments: We would like to acknowledge the support given by all the technical staff of the Department of the Technical Cybernetic department for their fruitful discussion.

Conflicts of Interest: The authors declare no conflict of interest.

\section{References}

1. Almeida, R.V.; Xu, Q.; Barrios, C.A.; Lipson, M. Guiding and confining light in void nanostructure. Opt. Lett. 2004, 29, 1209-1211. [CrossRef] [PubMed]

2. Degtyarev, S.A.; Butt, M.A.; Khonina, S.N.; Skidanov, R.V. Modelling of $\mathrm{TiO} 2$ based slot waveguides with high optical confinement in sharp bends. In Proceedings of the International Conference on Computing, Electronic and Electrical Engineering (ICECube), Quetta, Pakistan, 11-12 April 2016; pp. 10-13. [CrossRef]

3. Robinson, J.T.; Chen, L.; Lipson, M. On-chip gas detection in silicon optical microcavities. Opt. Express 2008, 16, 4296-4301. [CrossRef] [PubMed]

4. Khonina, S.N.; Kazanskiy, N.L.; Butt, M.A. Evanescent field ratio enhancement of a modified ridge waveguide structure for methane gas sensing application. IEEE Sens. J. 2020. [CrossRef]

5. Claes, T.; Molera, J.G.; De Vos, K.; Schacht, E.; Baets, R.; Bienstman, P. Label-free biosensing with a slot-waveguide-based ring resonator in silicon on insulator. IEEE Photonics J. 2009, 1, 197-204. [CrossRef]

6. Rytov, S.M. Electromagnetic properties of a finely stratified medium. Sov. Phys. JETP 1956, 2, 466-475.

7. Kanamori, Y.; Roy, E.; Chen, Y. Antireflection sub-wavelength gratings fabricated by spin-coating replication. Microelectron. Eng. 2005, 78-79, 287-293. [CrossRef]

8. Czyszanowski, T.; Gebski, M.; Dems, M.; Wasiak, M.; Sarzala, R.; Panajotov, K. Subwavelength grating as both emission mirror and electrical contact for VSCELs in any material system. Sci. Rep. 2017, 7, 40348. [CrossRef]

9. Mateus, C.F.R.; Huang, M.C.Y.; Chen, L.; Chang-Hasnain, C.J.; Suzuki, Y. Broad-band mirror (1.12-1.62 $\mu \mathrm{m})$ using a subwavelength grating. IEEE Photonics Technol. Lett. 2004, 16, 1676-1678. [CrossRef] 
10. Nambiar, S.; Selvaraja, S.K. Subwavelength grating fiber chip coupler in SOI with enhanced bandwidth and efficiency. In Proceedings of the 12th International Conference on Fiber Optics and Photonics, Kharagpur, India, 13-16 December 2014. [CrossRef]

11. Nambiar, S.; Sethi, P.; Selvaraja, S.K. Grating-Assisted fiber to chip coupling for SOI photonic circuits. Appl. Sci. 2018, 8, 1142. [CrossRef]

12. Yan, H.; Xu, X.; Chung, C.-J.; Subbaraman, H.; Pan, Z.; Chakravarty, S.; Chen, R.T. One dimensional photonic crystal slot waveguide for silicon-organic hybrid electro-optic modulators. Opt. Lett. 2016, 41, 5466-5469. [CrossRef]

13. Hogan, B.; Lewis, L.; McAuliffe, M.; Hegarty, S.P. Mid-infrared optical sensing using sub-wavelength gratings. Opt. Express 2019, 27, 3169-3179. [CrossRef] [PubMed]

14. Butt, M.A.; Kazanskiy, N.L.; Khonina, S.N. Model characteristics of refractive index engineered hybrid plasmonic waveguide. IEEE Sens. J. 2020. [CrossRef]

15. Yan, H.; Huang, L.; Xu, X.; Chakravarty, S.; Tang, N.; Tian, H.; Chen, R.T. Unique surface sensing property and enhanced sensitivity in microring resonator biosensors based on subwavelength grating waveguides. Opt. Express 2016, 24, 29724-29733. [CrossRef] [PubMed]

16. Cheng, Z.; Chen, X.; Wong, C.Y.; Xu, K.; Tsang, H.K. Apodized focusing subwavelength grating couplers for suspended membrane waveguides. Appl. Phys. Lett. 2012, 101, 101104. [CrossRef]

17. Huang, X.; Chen, G.; Zhou, W.; Huang, X. Cm-level photonic-crystal-like subwavelength waveguide platform with high integration density. Appl. Sci. 2019, 9, 3410. [CrossRef]

18. Xu, Y.; Bai, P.; Zhou, X.; Akimov, Y.; Png, C.E.; Ang, L.-K.; Knoll, W.; Wu, L. Optical refractive index sensors with plasmonic and photonic structures: Promising and inconvenient truth. Adv. Opt. Mater. 2019, 7, 1801433. [CrossRef]

19. Kazanskiy, N.L.; Khonina, S.N.; Butt, M.A. Plasmonic sensors based on Metal-insulator-metal waveguides for refractive index sensing applications: A brief review. Physica. E 2020, 117, 113798. [CrossRef]

20. Li, C.; Qiu, J.; Ou, J.-Y.; Liu, Q.H.; Zhu, J. High-sensitivity refractive index sensors using coherent perfect absorption on graphene in the Vis-NIR region. ACS Appl. Nano Mater. 2019, 2, 3231-3237. [CrossRef]

21. Gerislioglu, B.; Dong, L.; Ahmadivand, A.; Hu, H.; Nordlander, P.; Halas, N.J. Monolithic Metal Dimer-on-Film structure: New plasmonic properties introduced by the underlying metal. Nano Lett. 2020, 20, 2087-2093. [CrossRef]

22. Butt, M.A.; Khonina, S.N.; Kazanskiy, N.L. Modelling of rib channel waveguides based on silicon-on-sapphire at $4.67 \mu \mathrm{m}$ wavelength for evanescent field gas absorption sensor. Optik 2018, 168, 692-697. [CrossRef]

23. Butt, M.A.; Degtyarev, S.A.; Khonina, S.N.; Kazanskiy, N.L. An evanescent field absorption gas sensor at mid-IR $3.39 \mu \mathrm{m}$ wavelength. J. Mod. Opt. 2017, 64, 1892-1897. [CrossRef]

24. Fard, S.T.; Donzella, V.; Schmidt, S.A.; Flueckiger, J.; Grist, S.M.; Fard, P.T.; Wu, Y.; Bojko, R.J.; Kwok, E.; Jaeger, N.A.; et al. Performance of ultra-thin SOI-based resonators for sensing applications. Opt. Express 2014, 22, 14166-14179. [CrossRef]

25. Weissman, Z.; Hendel, I. Analysis of periodically segmented waveguide mode expanders. J. Lightw. Technol. 1995, 13, 2053-2058. [CrossRef]

26. Barrios, C.A. Optical slot-waveguide based biochemical sensors. Sensors 2009, 9, 4751-4765. [CrossRef] [PubMed]

27. Schmidt, S.; Flueckiger, J.; Wu, W.; Grist, S.M.; Fard, S.T.; Donzella, V.; Khumwan, P.; Thompson, E.R.; Wang, Q.; Kulik, P.; et al. Improving the performance of silicon photonic rings, disks, and Bragg gratings for use in label-free biosensing. SPIE Proc. 2014, 9166, 91660M.

28. Flueckiger, J.; Schmidt, S.; Donzella, V.; Sherwali, A.; Ratner, D.M.; Chrostowski, L.; Cheung, K.C. Sub-wavelength grating for enhanced ring resonator biosensor. Opt. Express 2016, 24, 15672-15686. [CrossRef]

29. Chrostowski, L.; Grist, S.; Flueckiger, J.; Shi, W.; Wang, X.; Ouellet, E.; Yun, H.; Webb, M.; Nie, B.; Liang, Z.; et al. Silicon photonic resonator sensors and devices. In Proceedings of the Presented at SPIE LASE (International Society for Optics and Photonics), San Francisco, CA, USA, 21-26 January 2012; p. 823620.

30. Wang, X.; Flueckiger, J.; Schmidt, S.; Grist, S.; Fard, S.T.; Kirk, J.; Doerfler, M.; Cheung, K.C.; Ratner, D.M.; Chrostowski, L.; et al. A silicon photonic biosensor using phase-shifted Bragg gratings in slot waveguide. J. Biophotonics 2013, 6, 821-828. 
31. Caroselli, R.; Ponce-Alcántara, S.; Quilez, F.P.; Sánchez, D.M.; Morán, L.T.; Barres, A.G.; Bellieres, L.; Bandarenka, H.; Girel, K.; Bondarenko, V.; et al. Experimental study of the sensitivity of a porous silicon ring resonator sensor using continuous in-flow measurements. Opt. Express 2017, 25, 31651-31659. [CrossRef] [PubMed]

32. Barrios, C.A.; Gylfason, K.B.; Sánchez, B.; Griol, A.; Sohlström, H.; Holgado, M.; Casquel, R. Slot waveguide biochemical sensor. Opt. Lett. 2007, 32, 3080-3082. [CrossRef] [PubMed]

33. Wang, Y.; Gao, B.; Zhang, K.; Yuan, K.; Wan, Y.; Xie, Z.; Xu, X.; Zhang, H.; Song, Q.; Yao, L.; et al. Refractive index sensor based on leaky resonant scattering of single semiconductor nanowire. ACS Photonics 2017, 4, 688-694. [CrossRef]

34. Xu, D.X.; Vachon, M.; Densmore, A.; Ma, R.; Janz, S.; Delâge, A.; Lapointe, J.; Cheben, P.; Schmid, J.H.; Post, E.; et al. Real-time cancellation of temperature induced resonance shifts in SOI wire waveguide ring resonator label-free biosensor arrays. Opt. Express 2010, 18, 22867-22879. [CrossRef] [PubMed]

35. TalebiFard, S.; Schmidt, S.; Shi, W.; Wu, W.; Jaeger, N.A.; Kwok, E.; Ratner, D.M.; Chrostowski, L. Optimized sensitivity of silicon-on-insulator (SOI) strip waveguide resonator sensor. Biomed. Opt. Express 2017, 8, 500-511. [CrossRef] [PubMed]

36. Hoste, J.-W.; Werquin, S.; Claes, T.; Bienstman, P. Conformational analysis of proteins with a dual polarisation silicon microring. Opt. Express 2014, 22, 2807-2820.

37. Butt, M.A.; Khonina, S.N.; Kazanskiy, N.L. A highly sensitive design of subwavelength grating double-slot waveguide microring resonator. Laser Phys. Lett. 2020, 17, 076201. [CrossRef]

(C) 2020 by the authors. Licensee MDPI, Basel, Switzerland. This article is an open access article distributed under the terms and conditions of the Creative Commons Attribution (CC BY) license (http://creativecommons.org/licenses/by/4.0/). 